DOI $10.35381 / \mathrm{cm} . v 6 \mathrm{i} 10.232$

\title{
Aprendizaje centrado en el estudiante desde la planificación en investigación
}

\author{
Student-centered learning from research planning \\ Myrian Liceth Mendoza Moreira \\ Mendozamoreira12@gmail.com \\ Pontificia Universidad Católica del Ecuador, Quito \\ Ecuador \\ https://orcid.org/0000-0001-8984-1145 \\ Maria Rodríguez Gámez \\ Taliangel270557@gmail.com \\ Universidad Tecnica de Manabí, Portoviejo \\ Ecuador \\ https://orcid.org/0000-0003-3178-0946
}

Recibido: 15 de julio de 2019

Aprobado: 15 de agosto de 2019

\section{RESUMEN}

La investigación analiza el aprendizaje centrado en el estudiante desde la planificación en investigación con la finalidad de conocer cómo se desarrolla este enfoque en las instituciones educativa del Ecuador. Se abordó un tipo de investigación descriptiva, entre los principales resultados se tiene que en este enfoque toma un rol protagónico que permite interactuar con sus compañeros en proporción de construir una comunidad de aprendizaje, siendo esto propicio desde la perspectiva de la sociedad del conocimiento donde se requiere de estudiantes críticos, reflexivos, desde la continua transformación del conocimiento, es necesario transcender la visión de enseñanza hacia un aprendizaje cooperativo en donde se construyen alternativas sustentables para el crecimiento humano en concordancia con las políticas educativas que requieren de una escuela activa y protagónica como organización generadora de posibilidades de convivencia basada en el respeto y dialogo, contribuyendo a formar ciudadanos que se enfrenten a los retos sociales con responsabilidad.

Descriptores: Educación experimental; Innovación educacional; Tendencia educacional; Prospección educacional. 


\begin{abstract}
The research analyzes student-centered learning from research planning in order to know how this approach develops in the educational institutions of Ecuador. A type of descriptive research was approached, among the main results is that in this approach it takes a leading role that allows interacting with peers in proportion to build a learning community, this being conducive from the perspective of the knowledge society where It requires critical, reflective students, from the continuous transformation of knowledge, it is necessary to transcend the vision of teaching towards cooperative learning where sustainable alternatives for human growth are constructed in accordance with the educational policies that require an active and leading school as organization that generates opportunities for coexistence based on respect and dialogue, contributing to educate citizens who face social challenges with responsibility.
\end{abstract}

Descriptors: Educational experiments; Educational innovations; Educational trends; Educational forecasting.

\title{
INTRODUCCIÓN
}

El aprendizaje centrado en el estudiante, es una vertiente que ha tenido auge desde las perspectivas constructivistas y socio críticas que han permitido establecer nuevos modelos de aprendizaje, donde el estudiante aprende en la medida que socializa en su entorno, recogiendo experiencias, vivencias, traducidas en conocimientos pertinentes de ser abordados no solo en la escuela, sino, en la dinámica social donde se desarrolla la persona, así se encuentra la investigación como actividad cotidiana del estudiante, generando una continua dialéctica que le permite acercarse al fenómeno de estudio desde sus propias motivaciones, esto implica considerar la planificación desde la vertiente de Colina Ysea (2015), al plantear que:

La planificación docente, se convierte en una herramienta fundamental de acción para organizar y sistematizar el aprendizaje, en atención al contexto social, el diagnóstico de las necesidades, los estadios cognitivos, prioridades e intereses de los educandos, como elementos que se articulan en la enseñanza de manera significativa; vivencial en la estructura cognitiva del educando (p. 191).

De ese modo, se desmonta la matriz mecanicista del aprendizaje, siendo posible articular desde el accionar docente un modelo educativo donde se planifique teniendo 
como centro la investigación como factor esencial de la generación de conocimientos, no solo dentro de las aulas, sino, en la cotidianidad del estudiante, esto hace posible conjugar la generación de un sujeto activo desde la perspectiva cognoscente en procura de cooperar en la germinación de su propio aprendizaje, siendo el docente un asesor que acompaña el proceso educativo.

El estudiante en este enfoque toma un rol protagónico que permite interactuar con sus compañeros en proporción de construir una comunidad de aprendizaje, siendo esto propicio desde la perspectiva de la sociedad del conocimiento donde se requiere de estudiantes críticos, reflexivos, desde la continua transformación del conocimiento, Martínez (2006, p. 246), plantea que el rol docente en este sentido debe "consistir en una postura exploratoria acerca de la compleja, rica y dinámica vida del aula, alejada, por tanto, de la actitud simple técnico que aplica rutinas preestablecidas a problemas estandarizados".

Lo cual implica transcender el estatus quo de la planificación tradicional en donde se transmite contenidos pre concebidos, sin tener en cuenta los intereses del estudiante en función de su contexto social o hábitat, lo cual coarta la creatividad e innovación, promoviéndose una educación desarticulada de la pertinencia social, sobre todo cuando el mundo transita al 2030 a una agenda sostenible, siendo necesario educar en complejidad de saberes, requiriéndose la formación de un investigador en razón de cooperar en la construcción de una mejor sociedad, para lograr este propósito, Stenhouse (1975), citado en Elliot (2000), considera la actividad de la investigación educativa en el aula, debe estar centrada en el diálogo constante, en esa interacción y socialización entre los actores educativos y en la revisión de sus propias acciones, originando al pensamiento crítico y reflexivo. En este sentido, Martínez (2006), considera la planificación docente desde la investigación, no se puede observar como algo rígido, paso por paso, procedimientos científicos preestablecidos, sino más bien como un proceso cíclico contextualizado a cada realidad educativa. 
Por consiguiente, el aprendizaje centrado en el estudiante, transciende la visión educativa donde el docente transmite protagónicamente sus conocimientos, siendo el estudiante un ente pasivo, en este sentido, el estudiante toma protagonismo, siendo quien desarrolla con primacía los conocimientos, constituyéndose en autónomo e independiente en la búsqueda del conocimiento, siendo el docente un asesor que acompaña el proceso.

Desde el punto de vista de la psicología educativa, se puede hacer mención del enfoque centrada en el cliente de Carls Roger, donde la persona que acude a la terapia toma el rol protagónico, responsabilizándose de sus acciones y decisiones, siendo el terapeuta un guía que acompaña el proceso, esta metáfora es transferida al aprendizaje centrado al estudiante, aunado a la aparición de las TIC como perspectiva educativa, la cual requiere de un estudiante activo en la construcción del conocimiento, ambos escenarios permiten tener un punto de referencia para no conjugar sobre la inexistencia teórica el enfoque centrado en el estudiante.

En el Ecuador en los últimos años se ha propuesto la transformación de la educación, en una propuesta inclusiva y de calidad para el buen vivir, para esto se requiere la generación de alternativas pedagógicas al modelo de enseñanza tradicional, siendo el aprendizaje centrado en el estudiante una alternativa viable que permite además conjugar la presencialidad con la virtualidad en aras de que el estudiante adopte una postura autónoma en la construcción del conocimiento, por consiguiente la actual investigación analiza el aprendizaje centrado en el estudiante desde la planificación en investigación con la finalidad de conocer cómo se desarrolla este enfoque en las instituciones educativa del Ecuador.

\section{DESARROLLO}

\section{Aprendizaje centrado en el estudiante}

Desde esta perspectiva el estudiante es el protagonista del aprendizaje, Macías (2017), plantea que: 
El estudiante debe ser protagonista de su propio aprendizaje y debe empoderarse y comprometerse con la actividad intelectual necesaria para asumir la construcción del conocimiento. Debe ser capaz de trabajar en equipo, aprendiendo a argumentar, a resolver problemas y a respetar las ideas de otros, pues es en la interacción en donde se construye una actitud ante el conocimiento, buscando información y comprometiéndose a la resolución de problemas reales y de su medio más cercano. (p. 1).

Esto modelo de aprendizaje, busca que el estudiante no solo genere procesos cognitivos, sino, procedimentales que le permitan construir conocimientos en contexto a su interacción social, construyéndose la posibilidad de interactuar con sus compañeros en la cooperación de la construcción de nuevos conocimientos. Esto transciende la educación tradicional, Kembel (2016), explica:

Si echamos la vista atrás, nuestro sistema educativo se ha basado en transferir a los estudiantes lo que sabemos y la forma de hacerlo son las clases magistrales. Con los exámenes se evalúa si los alumnos contestan o no lo correcto. Ese método ya no sirve. No sabemos los trabajos que existirán dentro de dos años y desconocemos lo que los jóvenes tendrán que resolver. En el mundo actual, todo cambia deprisa y hay que entrenar a las mentes para saber reaccionar frente a la incertidumbre. (p. 2).

El aprendizaje centrado en el estudiante, le permite formarse en competencias, aunado que le permite proyectar su vida para construir mediante un proyecto, la posibilidad de estar en concordancia con las exigencias socio laborales de un mercado competitivo y la vez innovador, factores que requieren de la creatividad como eslabón para configurar una mayor posibilidad de ser efectivo y exitoso, siendo necesario comprender que se requiere de mayor horizontalidad en la educación, Maya, Aldana \& Isea (2019), plantean que:

La estructura piramidal, no sólo de esta institución sino de casi todas en el ámbito nacional concibe a las personas como un recurso y no como seres de creatividad, limitando de esta manera la óptima gestión y liderazgo en la institución. (p. 127). 


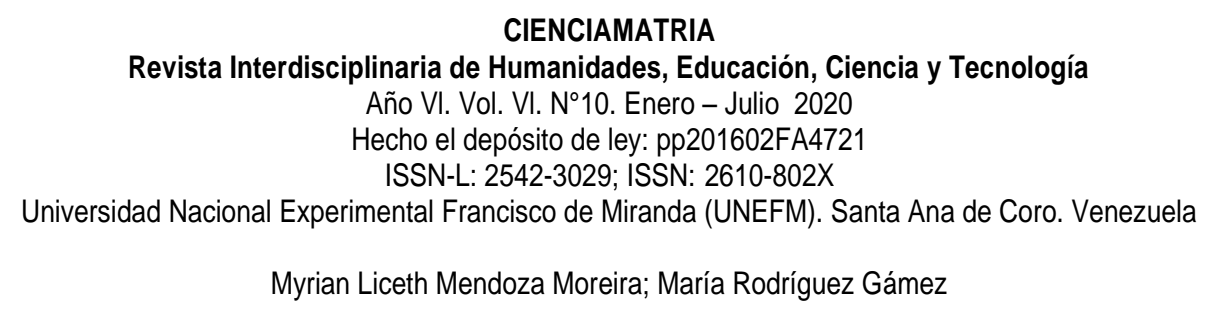

Esto involucra que estudiantes con sus pares y docentes, podrán tener mejor comunicación, constituyéndose equipos de trabajo que pueden ser la base para conformar proyectos de investigación, donde cada miembro aporte a la construcción significativamente del mismo, en este sentido, el Instituto Tecnológico y de Estudios Superiores de Monterrey (2018), complementa al indicar:

\section{Rol del estudiante}

1. Analizar situaciones reales, complejas y retadoras presentadas por el profesor

2. Buscar, estudiar y aplicar información de diversas fuentes (Internet, Biblioteca Digital, biblioteca del campus, textos, artículos, consultas a expertos de organizaciones y empresas) para ofrecer soluciones fundamentadas.

3. Compartir las soluciones con los miembros del grupo, buscando entre todos, de forma colaborativa, la solución más viable.

4. Utilizar las tecnologías de la información para aprender, investigar, exponer e interactuar con el profesor y sus compañeros.

5. Consultar al profesor y a otros expertos para pedir orientación cuando lo necesita.

6. Participar en la organización y administración del proceso compartiendo responsabilidades con sus compañeros.

7. Participar en sesiones de grupo para reflexionar sobre el proceso, los resultados logrados y proponer juntos soluciones de mejora bajo la guía del profesor.

\section{Rol del profesor}

1. Sigue fungiendo como experto en la materia que imparte, la cual conoce profunda y ampliamente, y se espera que como tal, aporte su experiencia y conocimientos para orientar, ampliar, enriquecer y clarificar los conocimientos que el alumno va construyendo a través de sus actividades. 


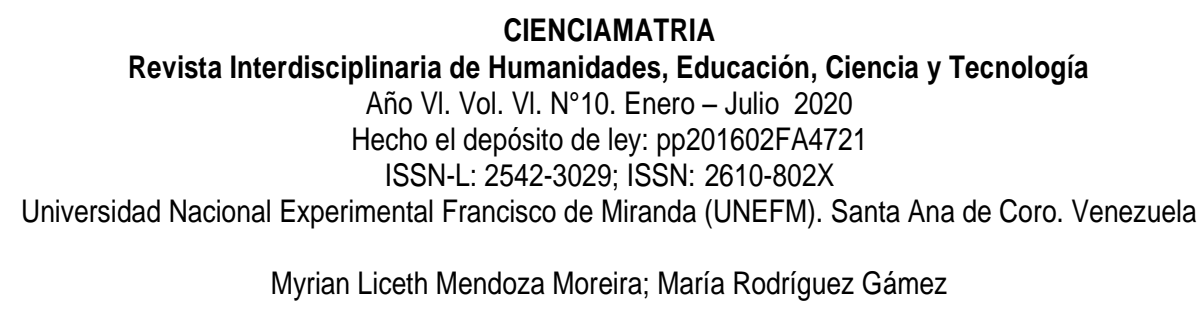

2. Explora e investiga situaciones de la vida real, relacionadas con los contenidos del curso, y las presenta a los alumnos en forma de casos, problemas o proyectos.

3. Planea, diseña y administra el proceso de aprendizaje y utiliza una plataforma tecnológica apropiada para documentar el curso y ponerlo a disposición del alumno, a fin de que sepa de antemano, qué se espera de él durante el curso y cómo será evaluado.

4. Dispone los espacios físicos de manera que se faciliten las conductas requeridas. El mobiliario, por ejemplo, debe estar organizado para que fluya el diálogo entre los alumnos y se logre una discusión efectiva.

5. Crea una atmósfera de trabajo que permita la apertura, la motivación y la libre expresión de los alumnos, y en la cual éstos sientan seguridad y respeto a su persona cuando hacen contribuciones al grupo.

6. Facilita el proceso de aprendizaje propiciando las condiciones adecuadas: selecciona las mejores experiencias, estimula con preguntas clave el pensamiento del alumno para que profundice en el conocimiento y lo orienta para que supere las dificultades y logre los objetivos de aprendizaje. Para cumplir con esta función, el profesor debe mantener una relación continua y personalizada con cada alumno.

7. Utiliza las herramientas tecnológicas para que el alumno tenga acceso a información actualizada a través de Internet y de la Biblioteca Digital; hace uso del correo electrónico para mantener una comunicación abierta con los alumnos, independientemente del lugar en el que se encuentre y puede así, ofrecer una asesoría oportuna; mantiene al grupo de estudiantes en interacción continua en espacios virtuales, donde puede también registrar sus contribuciones y estar accesible para los miembros del grupo.

8. Evalúa de forma permanente el desempeño del alumno. Observa sus conductas y analiza sus contribuciones y trabajos, compara estos datos con los criterios o 
estándares establecidos previamente, identifica donde están los problemas e interviene ofreciendo el apoyo requerido.

9. Actúa como líder del grupo, motivando a los alumnos durante todo el proceso, consciente de que un alumno motivado trabaja con más facilidad, es más resistente a la fatiga y mantiene un esfuerzo contenido ante las dificultades.

10. Crea una auténtica comunidad de aprendizaje donde los alumnos se sienten parte de un grupo en el que todos hacen sinergia y se ayudan mutuamente, donde el alumno es el actor y el profesor ayuda pero no invade ni sustituye el trabajo del alumno.

11. Investiga en el aula de forma continua, y hace mejoras y reajustes al plan establecido si lo requiere, y documenta los resultados. Este proceso mantiene al profesor en una actitud de mejora permanente, le permite identificar las experiencias y las actividades más adecuadas y ponerlas a disposición de los demás profesores.

12. Enseña con el ejemplo. El profesor debe ser en todo momento portador de los valores y conductas que desea fomentar en sus alumnos, caracterizándose por vivir y actuar de forma congruente con los principios establecidos en la misión. Es también a través de esta influencia como va modelando el carácter del estudiante

\section{MÉTODO}

Se aplicó una investigación tipo descriptiva, definida por Arias (2009) como la investigación que "consiste en la caracterización de un hecho, fenómeno o supo con establecer su estructura o comportamiento" (p. 64). Se abordó desde un diseño no experimental, transeccional de campo. Hernández, Fernández, Baptista (2014) refieren al diseño no experimental como "los estudios que se realizan sin la manipulación deliberada de variables y en los que sólo se observan los fenómenos en su ambiente natural para después analizarlos" (p. 267). 
La población de acuerdo a Tamayo y Tamayo (2007), "Es la totalidad del fenómeno a estudiar donde las unidades de población poseen una característica común la cual se estudia y da origen a los datos de la investigación" (p. 114), la cantidad de población estuvo conformada por 47 estudiantes de instituciones educativas del Ecuador, a quienes se les aplicó un instrumento de cinco alternativas de repuestas con escalamiento de Likert.

\section{RESULTADOS}

Se presentan los resultados de la investigación, producto de la aplicación de la encuesta a la población objeto de estudio.

Rol de estudiante.

Cuadro 1.

\begin{tabular}{|c|c|c|c|c|c|c|c|c|c|}
\hline \multicolumn{2}{|c|}{ SIEMPRE } & \multicolumn{2}{c|}{$\begin{array}{c}\text { CASI } \\
\text { SIEMPRE }\end{array}$} & \multicolumn{2}{c|}{$\begin{array}{c}\text { ALGUNAS } \\
\text { VECES }\end{array}$} & \multicolumn{2}{c|}{$\begin{array}{c}\text { CASI } \\
\text { NUNCA }\end{array}$} & \multicolumn{2}{c|}{ NUNCA } \\
\hline FA & $\%$ & FA & $\%$ & FA & $\%$ & FA & $\%$ & FA & $\%$ \\
\hline 5 & 10 & 5 & 11 & 6 & 13 & 17 & 36 & 14 & 30 \\
\hline
\end{tabular}

La opción siempre tuvo una representación estadística del 10\%, así mismo la opción casi siempre un $11 \%$, la alternativa algunas veces $13 \%$ y las opciones casi nunca $36 \%$ y nunca el $30 \%$

Rol docente.

Cuadro 2.

\begin{tabular}{|c|c|c|c|c|c|c|c|c|c|}
\hline \multicolumn{2}{|c|}{ SIEMPRE } & \multicolumn{2}{c|}{$\begin{array}{c}\text { CASI } \\
\text { SIEMPRE }\end{array}$} & \multicolumn{2}{c|}{$\begin{array}{c}\text { ALGUNAS } \\
\text { VECES }\end{array}$} & \multicolumn{2}{c|}{$\begin{array}{c}\text { CASI } \\
\text { NUNCA }\end{array}$} & \multicolumn{2}{c|}{ NUNCA } \\
\hline FA & $\%$ & FA & $\%$ & FA & $\%$ & FA & $\%$ & FA & $\%$ \\
\hline 6 & 13 & 8 & 17 & 10 & 21 & 10 & 21 & 13 & 28 \\
\hline
\end{tabular}

La opción siempre tuvo una representación estadística del 13\%, así mismo la opción casi siempre un $17 \%$, la alternativa algunas veces $21 \%$ y las opciones casi nunca con un $21 \%$ y nunca $28 \%$. 


\section{DISCUSIÓN}

Al agruparse los resultados desde la perspectiva del rol del estudiante y del docente en el aprendizaje centrado en el estudiante, se percibe que este proceso no se está desarrollando efectivamente, lo cual implica que la investigación como proceso de planificación docente no se está ejecutando como cooperador del enfoque centrado en el estudiante, contradiciendo lo planteado por lanni Gómez (2017), al indicar:

La evaluación, por consiguiente, se ha de asumir desde una perspectiva donde la investigación y la reflexión son los ejes dinamizadores de dicho proceso. En este sentido, la evaluación se concibe como un elemento integrador y articulador del proceso educativo, es decir no se puede concebir separadamente de la enseñanza y el aprendizaje (p. 28).

De ese modo, el aprendizaje se conjuga en una interrelación compleja, donde la investigación se constituye en el medio para construir conocimientos desde la praxis investigativa, lo cual le permite al estudiante reflexionar constantemente sobre su actuación como persona, estudiante, investigador, pudiendo auto evaluarse para tomar decisiones que le permitan mejorar para crecer en un marco de flexibilidad, siendo esta una competencia transversal para la vida.

\section{CONCLUSIONES}

El enfoque centrado en el estudiante permite aprender para la vida, teniendo la investigación como un factor esencial que contribuye a ser veraz en la información que se consume, posibilitándose la generación de una personalidad critica - reflexiva, asumiendo las potencialidades y debilidades como una oportunidad para el crecimiento integral, producto de la reflexión por parte del estudiante, propiciándose la generación de conocimientos en pertinencia a los retos globales existentes, de ese modo, el estudiante se prepara para asumir con pertinencia un proyecto de vida apegado a la ética en concordancia con ser ciudadano en posibilidad de proponer soluciones a los diversos problemas colectivos de la sociedad. 
De lo que se trata el enfoque centrado en el estudiante, es transcender la visión de enseñanza hacia un aprendizaje cooperativo en donde se construyen alternativas sustentables para el crecimiento humano en concordancia con las políticas educativas que requieren de una escuela activa y protagónica como organización generadora de posibilidades de convivencia basada en el respeto y dialogo, contribuyendo a formar ciudadanos que se enfrenten a los retos sociales con responsabilidad y compromiso social.

\section{REFERENCIAS CONSULTADAS}

1. Arias, F. (2009). El Proyecto de Investigación. Guía para su elaboración. Caracas: Epísteme. Quinta Edición.

2. Colina Ysea, F. (2015). Planificación docente e investigación. Agentes articuladores de la gestión del conocimiento. Recuperado de http://www.fundacionkoinonia.com.ve/publicaciones/planificacion docente inves tigacion.pdf

3. Elliot, J. (2000). La investigación-acción en educación. Ediciones Morata, S.L. Cuarta edición. Madrid-España.

4. Hernández, Fernández y Baptista (2014). Metodología de la investigación. México, Mc Graw Hill Hispanoamericana. Hill Internacional

5. Ianni Gómez., L. (2017). MIRAMDA, una propuesta educativa emergente desde la investigación. Revista Arbitrada Interdisciplinaria Koinonía, 2(3), 9-30. Recuperado de http://fundacionkoinonia.com.ve/ojs/index.php/revistakoinonia/article/view/49/36

6. Instituto Tecnológico y de Estudios Superiores de Monterrey (2018). Un modelo centrado en el aprendizaje. Recuperado de http://sitios.itesm.mx/va/dide/modelo/libro/capitulos_espanol/pdf/cap_2.pdfhttp:// sitios.itesm.mx/va/dide/modelo/libro/capitulos_espanol/pdf/cap_2.pdf

7. Kembel, G. (2016). Se aprende haciendo y no escuchando a un profesor. Recuperado de https://elpais.com/economia/2016/01/22/actualidad/1453461456_561424.html 


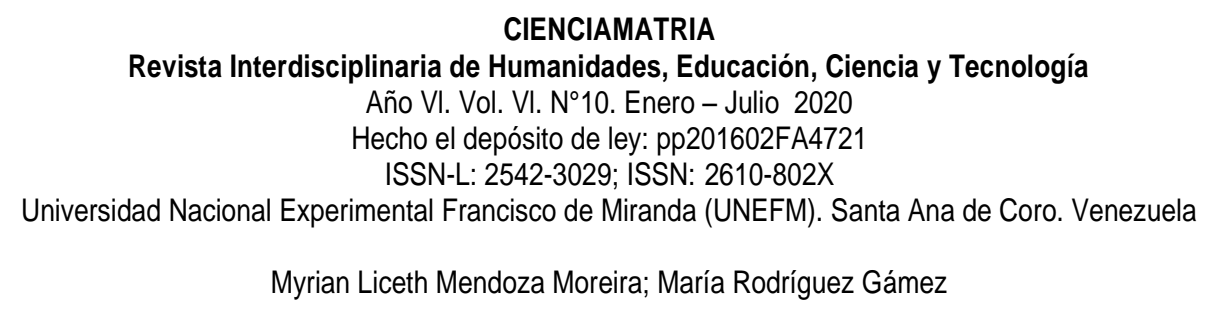

8. Macias, M. (2017). El estudiante como protagonista de su propio aprendizaje. Recuperado de https://ineverycrea.mx/comunidad/ineverycreamexico/recurso/elalumno-como-protagonista-de-su-propio/765cad1d-0696-43bb-9add$\underline{649 a 7 e 1 c 5650}$

9. Maya, E., Aldana Zavala, J., \& Isea Argüelles, J. (2019). Liderazgo Directivo y Educación de Calidad. CIENCIAMATRIA, 5(9), 114 - 129. Recuperado a partir de http://www.cienciamatriarevista.org.ve/index.php/cm/article/view/102

10.Martínez, M. (2006). Ciencias y Arte en la Metodología Cualitativa. Editorial Trillas. México.

11. Tamayo y Tamayo (2009). El proceso de investigación científica. Limusa. México.

\section{REFERENCES CONSULTED}

1. Arias, F. (2009). El Proyecto de Investigación. Guía para su elaboración. Caracas: Epísteme. Quinta Edición.

2. Colina Ysea, F. (2015). Planificación docente e investigación. Agentes articuladores de la gestión del conocimiento. Recuperado de http://www.fundacionkoinonia.com.ve/publicaciones/planificacion docente investi gacion.pdf

3. Elliot, J. (2000). La investigación-acción en educación. Ediciones Morata, S.L. Cuarta edición. Madrid-España.

4. Hernández, Fernández y Baptista (2014). Metodología de la investigación. México, Mc Graw Hill Hispanoamericana. Hill Internacional

5. Ianni Gómez., L. (2017). MIRAMDA, una propuesta educativa emergente desde la investigación. Revista Arbitrada Interdisciplinaria Koinonía, 2 (3), 9-30. Recuperado de http://fundacionkoinonia.com.ve/ojs/index.php/revistakoinonia/article/view/49/36

6. Instituto Tecnológico y de Estudios Superiores de Monterrey (2018). Un modelo centrado en el aprendizaje. Recuperado de http://sitios.itesm.mx/va/dide/modelo/libro/capitulos espanol/pdf/cap 2.pdfhttp://si tios.itesm.mx/va/dide/modelo/libro/capitulos espanol/pdf/cap 2.pdf 
Revista Interdisciplinaria de Humanidades, Educación, Ciencia y Tecnología

Año VI. Vol. VI. №10. Enero - Julio 2020

Hecho el depósito de ley: pp201602FA4721

ISSN-L: 2542-3029; ISSN: 2610-802X

Universidad Nacional Experimental Francisco de Miranda (UNEFM). Santa Ana de Coro. Venezuela

Myrian Liceth Mendoza Moreira; María Rodríguez Gámez

7. Kembel, G. (2016). Se aprende haciendo y no escuchando a un profesor. Recuperado de https://elpais.com/economia/2016/01/22/actualidad/1453461456 561424.html

8. Macías, M. (2017). El estudiante como protagonista de su propio aprendizaje. Recuperado de https://ineverycrea.mx/comunidad/ineverycreamexico/recurso/elalumno-como-protagonista-de-su-propio/765cad1d-0696-43bb-9add$\underline{649 a 7 e 1 c 5650}$

9. Maya, E., Aldana Zavala, J. e Isea Argüelles, J. (2019). Liderazgo Directivo y Educación de Calidad. CIENCIAMATRIA, 5 (9), 114 - 129. Recuperado de http://www.cienciamatriarevista.org.ve/index.php/cm/article/view/102

10. Martínez, M. (2006). Ciencias y Arte en la Metodología Cualitativa. Editorial Trillas. México

11. Tamayo y Tamayo (2009). El proceso de investigación científica. Limusa México. 